\title{
PHENOMENOLOGICAL MODELS AND ANIMATIONS OF WELDING AND THEIR IMPACT
}

\author{
Tarasankar DebRoy $^{1}$ \\ ${ }^{1}$ Professor of Materials Science and Engineering \\ 115 Steidle Building, The Penn State University, University Park, Pennsylvania, USA, 16802 \\ debroy@matse.psu.edu
}

Keywords: welding, phenomenological models, welding processes, metal structure

\begin{abstract}
Professor Robertson's recognized research on metallurgical thermodynamics and kinetics for over 40 years facilitated the emergence of rigorous quantitative understanding of many complex metallurgical processes. The author had the opportunity to work with Professor Robertson on liquid metals in the 1970s. This paper is intended to review the advances in the quantitative understanding of welding processes and weld metal attributes in recent decades. Over this period, phenomenological models have been developed to better understand and control various welding processes and the structure and properties of welded materials. Numerical models and animations of melting, solidification and the evolution of micro and macro-structural features will be presented to critically examine their impact on the practice of welding and the underlying science.
\end{abstract}

